\title{
Suppression of acetylpolyamine oxidase by selected AP-1 members regulates DNp73 abundance: mechanistic insights for overcoming DNp73-mediated resistance to chemotherapeutic drugs
}

\author{
W Bunjobpol ${ }^{1}$, I Dulloo $^{1}$, K Igarashi ${ }^{2}$, N Concin ${ }^{3}$, K Matsuo ${ }^{4}$ and K Sabapathy, ${ }^{*, 1,5,6}$
}

Enhanced resistance to chemotherapy has been correlated with high levels of Delta-Np73 (DNp73), an anti-apoptotic protein of the p53 tumor-suppressor family which inhibits the pro-apoptotic members such as $\mathrm{p} 53$ and TAp73. Although genotoxic drugs have been shown to induce DNp73 degradation, lack of mechanistic understanding of this process precludes strategies to enhance the targeting of DNp73 and improve treatment outcomes. Antizyme (Az) is a mediator of ubiquitin-independent protein degradation regulated by the polyamine biosynthesis pathway. We show here that acetylpolyamine oxidase (PAOX), a catabolic enzyme of this pathway, upregulates DNp73 levels by suppressing its degradation via the Az pathway. Conversely, downregulation of PAOX activity by siRNA-mediated knockdown or chemical inhibition leads to DNp73 degradation in an Az-dependent manner. PAOX expression is suppressed by several genotoxic drugs, via selected members of the activator protein-1 (AP-1) transcription factors, namely C-Jun, JunB and FosB, which are required for stress-mediated DNp73 degradation. Finally, chemical- and siRNA-mediated inhibition of PAOX significantly reversed the resistant phenotype of DNp73-overexpressing cancer cells to genotoxic drugs. Together, these data define a critical mechanism for the regulation of DNp73 abundance, and reveal that inhibition of PAOX could widen the therapeutic index of cytotoxic drugs and overcome DNp73-mediated chemoresistance in tumors.

Cell Death and Differentiation (2014) 21, 1240-1249; doi:10.1038/cdd.2014.41; published online 11 April 2014

$p 73$ is a member of the p53 family of transcription factors and encodes two major forms of the protein: the full-length TAp73 and the amino-terminally truncated Delta-Np73 (DNp73). ${ }^{1}$ Similar to p53, transactivation competent TAp73 exerts tumor-suppressive activities via the regulation of apoptotic signaling and cell-cycle arrest. ${ }^{2}$ However, the DNp73 form, which lacks the $\mathrm{N}$-terminal transactivation domain, works as a dominant-negative inhibitor of both TAp73 and p53 and therefore inhibits their tumor-suppressor functions. ${ }^{3}$ It is noteworthy that DNp73 also possesses weak transcriptional activity and is able to transactivate several genes such as 14-3-3 and Gadd45, ${ }^{4}$ and was recently shown to induce the expression of anti-apoptotic proteins caspase-2S and hsp70, ${ }^{5,6}$ further illustrating its anti-apoptotic potential. Consistently, DNp73 ${ }^{-/-}$mouse embryonic fibroblasts were found to be sensitized to genotoxic stresses and showed enhanced p53-dependent apoptosis, indicating that DNp73 is a critical inhibitor of apoptosis. ${ }^{7}$

Not surprisingly, DNp73 expression is frequently elevated in many human cancers, including lung, neuroblastoma, colon, ovarian, liver, etc. ${ }^{8-10}$ which suggests a role in either promoting cellular growth or inhibiting apoptosis, as well as in affecting chemosensitivity. In the latter context, TAp73 and p53 are activated by a multitude of chemotherapeutic agents, ${ }^{11}$ linking their functions to the chemosensitivity of cancer cells. Thus, DNp73 overexpression would be one mechanism of inhibiting TAp73- and p53-dependent pro-apoptotic activity in response to chemotherapeutic drugs, leading to poor clinical outcome. ${ }^{12,13}$ Consistently, exposure to genotoxic signals leads to the upregulation of TAp73 and the downregulation of DNp73, ${ }^{14}$ hence allowing an effective apoptotic response to ensue. This indicates that the relative abundance of the two forms of $p 73$ could be an important determinant of cellular fate during stress response, thereby regulating chemosensitivity.

Owing to their homology, TAp73 and DNp73 protein stability are regulated by many common factors, including Itch, c-Abl, PML, NQO1, Cyclin G and others, via both ubiquitin-dependent and -independent mechanisms. ${ }^{15-19}$ However, we have previously shown that the selective

${ }^{1}$ Laboratory of Molecular Carcinogenesis, Division of Cellular and Molecular Research, Humphrey Oei Institute of Cancer Research, National Cancer Centre, 11, Hospital Drive, Singapore, Singapore; ${ }^{2}$ Amine Pharma Research Institute, Innovation Plaza at Chiba University, 1-8-15Inohana, Chiba, Japan; ${ }^{3}$ Department of Obstetrics and Gynecology, Innsbruck Medical University, Anichstrasse 35, Innsbruck, Austria; ${ }^{4}$ Department of Microbiology and Immunology, School of Medicine, Keio University, 35 Shinanomachi, Tokyo, Japan; ${ }^{5}$ Cancer and Stem Cell Biology Program, Duke-NUS Graduate Medical School, 8, College Road, Singapore, Singapore and ${ }^{6}$ Department of Biochemistry, National University of Singapore, 8, Medical Drive, Singapore, Singapore

${ }^{*}$ Corresponding author: Professor K Sabapathy, Laboratory of Molecular Carcinogenesis, Division of Cellular and Molecular Research, Humphrey Oei Institute of Cancer Research, National Cancer Centre, 11, Hospital Drive, Singapore 169610, Singapore. Tel: +65 6436 8349; Fax: +65 6226 5694; E-mail: cmrksb@nccs.com.sg Abbreviations: AP-1, activator protein-1; Az, Antizyme; DNp73, Delta-N p73; PAOX, polyamine oxidase; ODC, ornithine decarboxylase; SAMDC, S-adenosylmethionine decarboxylase; SPDS, spermidine synthase; SPMS, spermine synthase; SSAT, spermidine/spermine $N^{1}$-acetyltransferase; CHX, cycloheximide; NAsp, acetylspermine; PI, propidium iodide; ChIP, chromatin immunoprecipitation; SDM, site-directed mutagenesis

Received 06.8.13; revised 17.2.14; accepted 28.2.14; Edited by G Melino; published online 11.4.14 
degradation of DNp73 by genotoxic stresses occur through the ubiquitin-independent, polyamine-induced antizyme $(A z)$ pathway that is regulated by c-Jun, a member of the activator protein-1 (AP-1) family of transcription factors. ${ }^{20} \mathrm{Az} 1$ is a small inhibitory protein that binds to its target proteins and directs them to the proteasome for degradation. ${ }^{21,22}$ Az1 transcript exists with two overlapping open reading frames, and translation of a fully functional Az1 protein requires a unique +1 ribosomal frameshift mechanism, which is dependent on the level of polyamines, a group of cationic compounds such as putrescine, spermidine and spermine present in the cells $s^{21,22}$ (Figure 1a, lower panel).

The balance of polyamines in cells is controlled by several key enzymes, which either synthesize or breakdown the different polyamine by-products. Ornithine is converted into diamine putrescine by the rate-limiting enzyme ornithine decarboxylase (ODC). ${ }^{22} \mathrm{~A}$ second rate-limiting enzyme S-adenosylmethionine decarboxylase (SAMDC) is required for the synthesis of both triamine spermidine and tetramine spermine by spermidine synthase (SPDS) and spermine

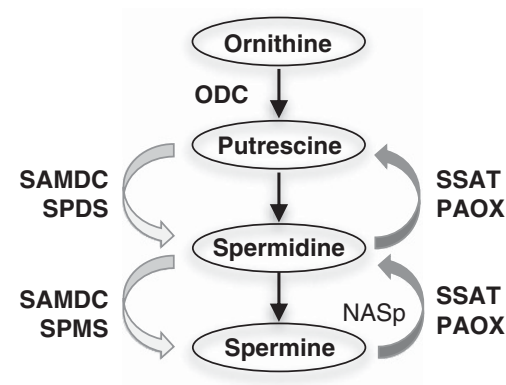

Polyamines

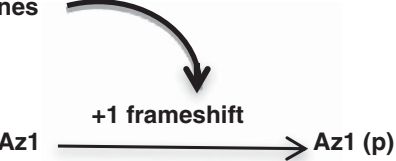

b

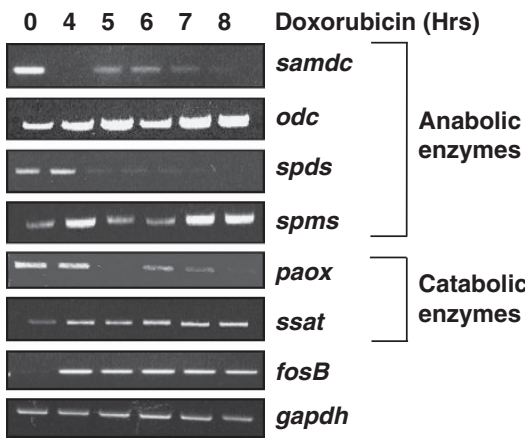

C

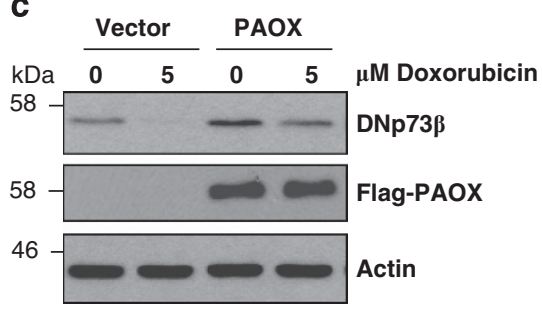

d
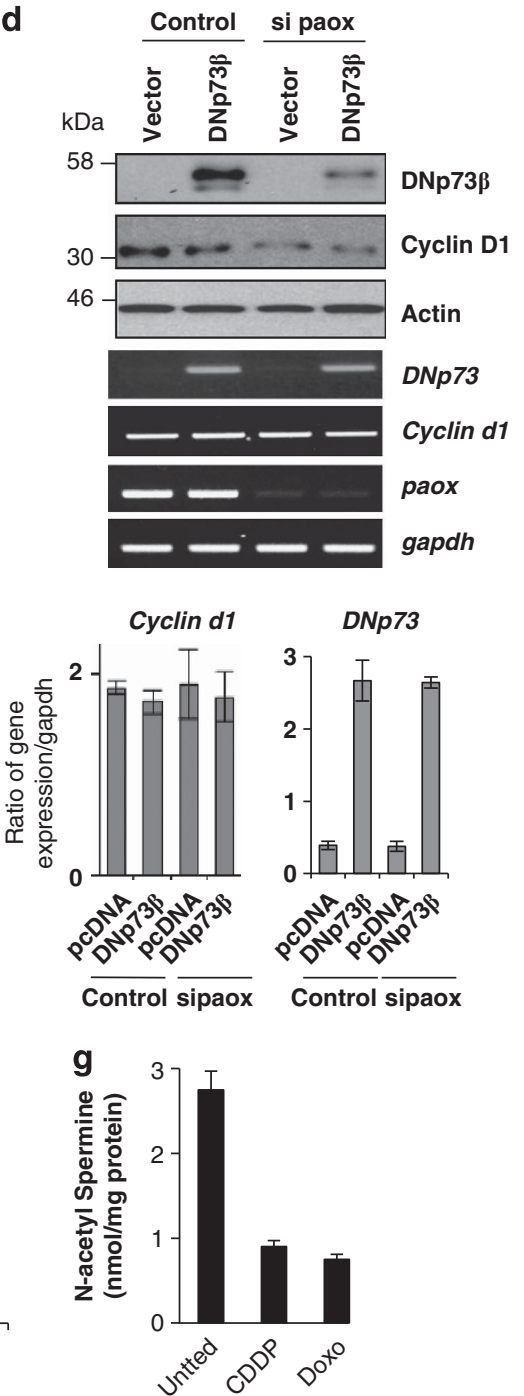

Figure 1 Polyamine catabolic enzyme paox regulates DNp73 stability. (a) Schematic showing the polyamines (putrescine, spermidine and spermine) biosynthesis pathway and the involvement of anabolic (ODC, SAMDC, SPDS and SPMS) and catabolic enzymes (PAOX and SSAT; upper panel) and the effects of polyamines on Az1 frameshifting process (lower panel). N-acetylspermine (NASp) is the intermediate in the catabolic conversion from spermine to spermidine. (b) Semi-quantitative RT-PCR analysis of the expression of enzymes in the polyamine biosynthesis pathway in p53 null $\mathrm{H} 1299$ cells exposed to doxorubicin ( $5 \mu \mathrm{M}$ ) for the indicated times. (c) Immunoblot analysis of steady-state DNp73 $\beta$ levels in H1299 cells transfected with or without Flag-tagged PAOX for $24 \mathrm{~h}$ followed by exposure to doxorubicin for $8 \mathrm{~h}$. Expression of exogenous PAOX and DNp73 was detected by anti-Flag and anti-p73 (GC15) antibodies, respectively. (d) Immunoblot and semi-quantitative RT-PCR (top and middle panels), and quantitative real-time PCR (lower bar charts) analysis of overexpressed DNp73 $\beta$ or endogenous cyclin D1 in H1299 cells was analyzed $48 \mathrm{~h}$ after siRNA-mediated silencing of paox. All values are means \pm S.D. of duplicate experiments, reflected by error bars. (e) $\mathrm{H} 1299$ cells inducibly expressing DNp $73 \beta$ were induced by doxycycline for $24 \mathrm{~h}$ before treatment with the PAOX inhibitor, MDL72527, at the indicated doses for $16 \mathrm{~h}$ and harvested for immunodetection. All experiments were repeated at least thrice and representative data are shown throughout. ( $\mathbf{f}$ and $\mathbf{g}$ ) Intracellular levels of spermine and putrescine were measured in H1299 cells (left bars) or HCT116 cells (right bars) without (Untted) or with cisplatin ( $25 \mu \mathrm{M} \mathrm{CDDP}$ ) or doxorubicin ( $5 \mu \mathrm{M}$ Doxo) treatment for $6 \mathrm{~h}$ by HPLC and the ratio (see Supplementary Figure 1D for raw data) is presented (f). The levels of the intermediate product in the conversion of spermine back to spermidine, NASp was also measured and depicted (g). All values are means \pm S.D. of triplicate experiments for $\mathbf{g}$ 
synthase (SPMS), respectively ${ }^{22}$ (Figure 1a, upper panel). Conversely, spermine can be converted back to spermidine and the latter to putrescine by catabolic enzymes spermidine/ spermine $N^{1}$-acetyltransferase (SSAT) and acetylpolyamine oxidase (PAOX); ${ }^{22}$ involving the intermediates $\mathrm{N}$-acetylspermine (NASp) and $\mathrm{N}$-acetylspermidine. Our previous study has shown that upon genotoxic insults, c-Jun regulates the levels of functional Az to degrade DNp73, but the exact mechanistic details remain unclear.

In addition to c-Jun, the AP-1 transcription factor family also includes JunB, JunD, c-Fos, FosB, Fra1 and Fra2. ${ }^{23}$ Through the formation of heterodimers or homodimers, the latter in the case of the Jun proteins, the AP-1 members regulate diverse physiological processes in response to a plethora of stimuli, such as DNA damage, growth factors and oncogenic stimuli. $^{24}$ Although AP-1 members can activate a set of common target genes, different AP-1 dimer compositions produce different biological outcomes, suggesting that they can also selectively regulate distinct sets of genes. ${ }^{25,26}$ In addition, some biological effects of AP-1 members are the result of their ability to repress gene expression, such as inhibition of $p 53$ by c-Jun. ${ }^{27}$

In this respect, we have investigated the mechanism of DNp73 regulation through the $A z$ pathway with reference to the specificity of AP-1 members in regulating this process. Our results demonstrate that unlike other AP-1 factors, only c-Jun, JunB and FosB are able to specifically repress the expression of polyamine catabolic enzyme paox (the gene encoding PAOX), highlighting the functional specificity of these AP-1 factors. This effect leads to the production of active $A z 1$ that consequently degrades DNp73. In addition, inhibition of PAOX in DNp73-overexpressing cancer cells abrogated their resistance to chemotherapeutic agents and reveals a possible avenue for effective targeted therapy in DNp73-overexpressing cancers in the presence of chemotherapeutic drugs.

\section{Results}

Polyamine catabolic enzyme PAOX positively regulates DNp73. We have utilized doxorubicin as a prototypic DNA-damaging chemotherapeutic agent to investigate the effects on the expression of the anabolic and catabolic enzymes that regulate polyamines levels in cells (Figure 1a). Treatment of p53 null H1299 cells with doxorubicin resulted in the transcriptional downregulation of anabolic enzymes samdc and spds and upregulation of odc and spms (Figure 1b). Although upregulation of odc and spms would equate to an increase in synthesis of polyamines, silencing of odc or spms did not prevent the stress-mediated degradation of DNp73 (Supplementary Figures 1 A and B). Similarly, although doxorubicin induced ssat expression, siRNAmediated silencing of this enzyme had no major effect on stress-mediated DNp73 degradation (Supplementary Figure 1B). However, downregulation of paox upon doxorubicin treatment was observed to occur concomitantly with the reduction of DNp73 $\beta$ protein (Supplementary Figure 1C), and exogenous expression of flag-tagged PAOX alone was able to significantly increase the steady-state levels of DNp73 $\beta$ after doxorubicin treatment (Figure 1c). Moreover, although doxorubicin treatment led to a reduction of DNp73 $\beta$, this effect was reduced in the presence of PAOX (Figure 1c). To further explore the role of PAOX in regulating DNp73 abundance, we silenced paox expression, which resulted in a marked decrease in DNp73 $\beta$ steady-state levels (Figure 1d). Similar effect was observed on endogenous cyclin D1, another known target of the polyamine-Az degradation pathway (Figure 1d), with the mRNA of both DNp73 and cyclin D1 remaining unaffected by paox silencing (Figure 1d lower panel). Furthermore, treatment of cells that inducibly express DNp73 $\beta$ with a known chemical inhibitor of PAOX, MDL72527, ${ }^{28}$ led to a decrease in DNp73 levels in a dose-dependent manner (Figure 1e).

We further evaluated the effects of the chemotherapeutic agents on the polyamine pathway by determining the levels of polyamines upon doxorubicin or cisplatin treatment (Supplementary Figure 1D). Although spermine levels were generally unaltered, levels of putrescine were reduced upon treatment (Supplementary Figure 1D), resulting in an increase in the ratio of spermine to putrescine (Figure 1f). Importantly, the levels of NASp were significantly reduced after drug treatment (Figure 1g), consistent with the reduction of paox by treatment with these agents. These data together, demonstrate that the polyamine catabolic enzyme paox that is downregulated upon genotoxic stress is critical in functioning as a regulator of DNp73 levels.

PAOX is regulated by selected AP-1 members. Treatment of cells with doxorubicin or cisplatin led to the downregulation of paox, which paralleled an increase in the expression of AP-1 factors C-Jun and FosB, concomitant to an increase of processed $A z[A z 1(p)]$ (Figure 2a). We therefore examined whether $\mathrm{c}$-Jun and other AP-1 members are able to regulate paox expression. Overexpression of only c-Jun, JunB and FosB led to a dose-dependent downregulation of paox expression, whereas no significant effects were observed with c-Fos, Fra1 (Figure 2b) and JunD (data not shown). Conversely, silencing their expression resulted in increased basal paox expression as well as reduced its downregulation by stress (Figure 2c), thus confirming the requirement of c-Jun, JunB and FosB for the downregulation of paox upon doxorubicin stress.

We next investigated whether all three AP-1 transcription factors were able to repress paox expression by directly acting on its promoter. For this purpose, we cloned a portion of the paox promoter $(-3045$ to -997$)$ predicted by TESS (Transcription Element Search System online software) to contain two potential AP-1-binding sites (indicated as P1 and P2 in Figure 2d upper panel) into a luciferase reporter construct. Overexpression of c-Jun and JunB led to a significant decrease, and FosB led to a marginal decrease in paox promoter activity (Figure 2d, lower left panel) and in parallel, upregulated collagenase promoter activity, a classical target of AP-1 transcription factors ${ }^{27}$ (Figure $2 d$, lower right panel). Members of the AP-1 transcription factors form heterodimers with each other to regulate many of their target genes $^{25,27}$ and expectedly, AP-1 monomers that were tethered to force specific pairing of c-Jun/FosB and JunB/FosB ${ }^{26}$ suppressed paox or activated collagenase to a higher level relative to $\mathrm{c}$-Jun, JunB and FosB alone 
a

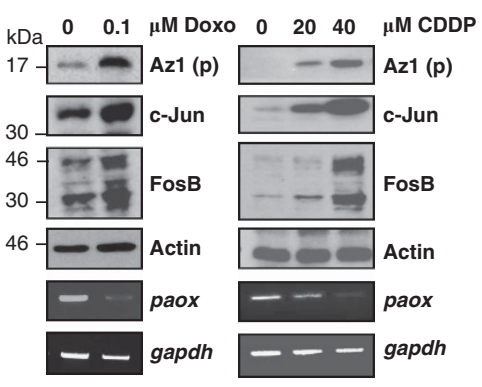

C

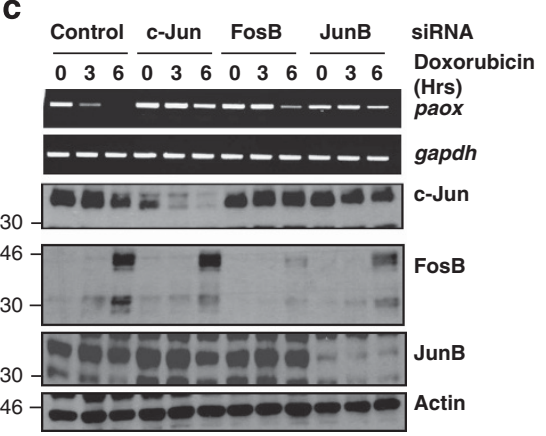

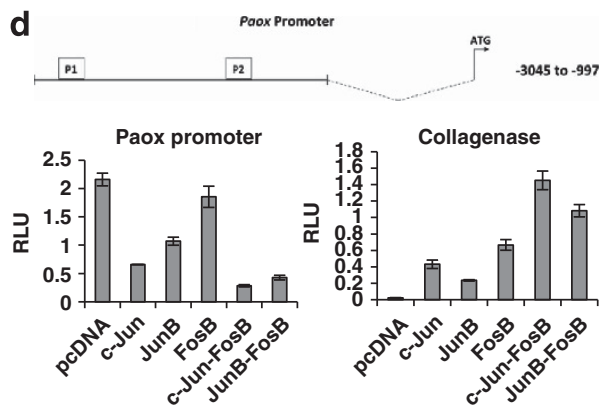

b
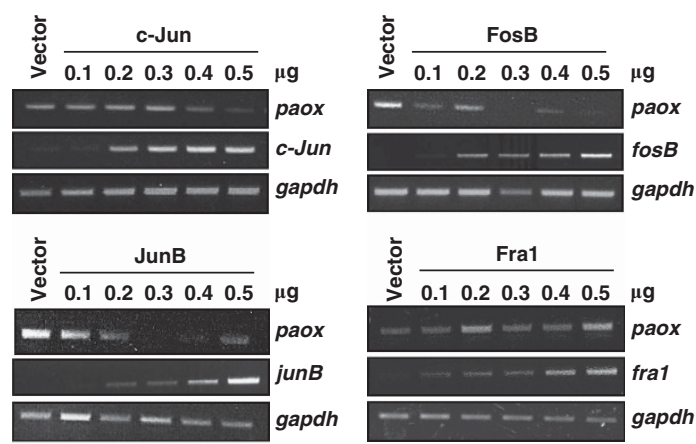

e
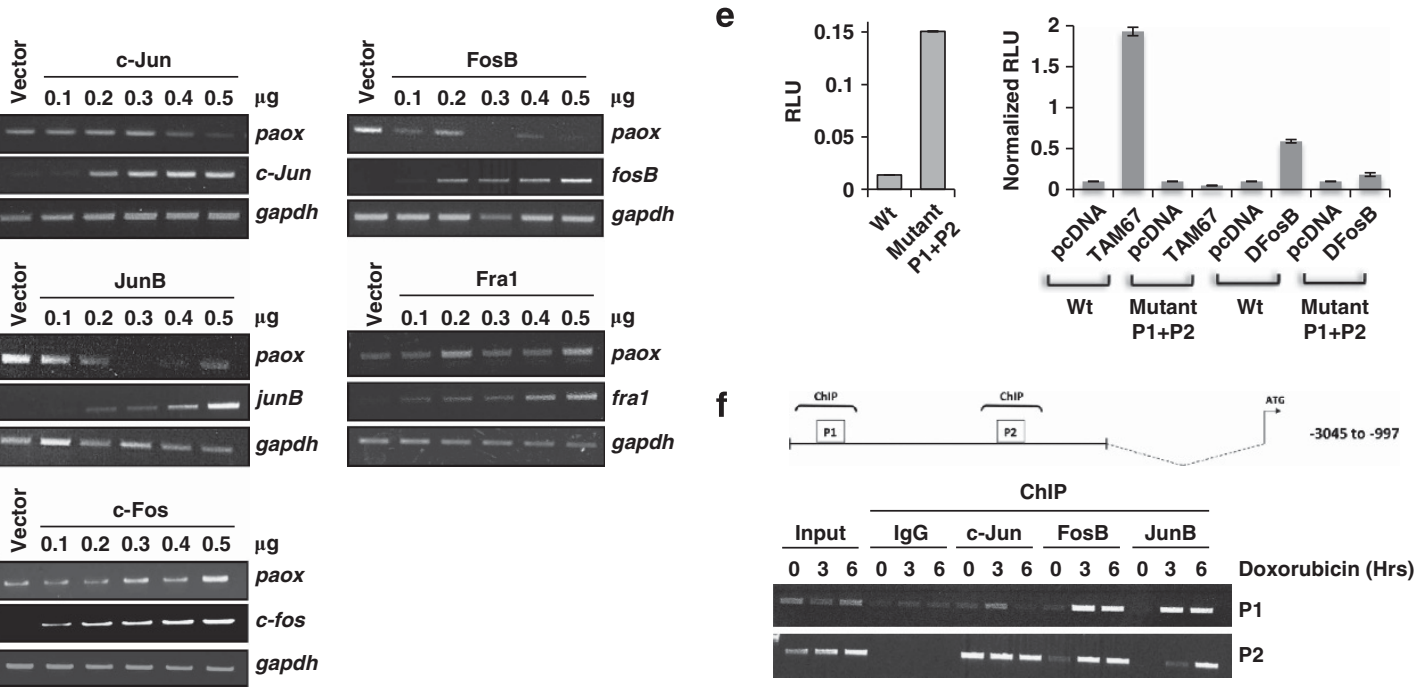

Figure 2 Polyamine oxidase (paox) expression is selectively regulated by AP-1 family members. (a) Expression of processed antizyme, Az1(p), and the indicated AP-1 members were analyzed by immunoblotting, and mRNA expression of paox was monitored by semi-quantitative RT-PCR, after exposure of H1299 cells to different concentration of doxorubicin (doxo) or cisplatin (CDPP), for $16 \mathrm{~h}$. (b) Expression of paox was determined by RT-PCR $24 \mathrm{~h}$ after transfection of increasing amounts of indicated AP-1 members in $\mathrm{H} 1299$ cells. (c) Effect of siRNA-mediated silencing of $c$-jun, fosB or junB on the expression of paox mRNA upon doxorubicin treatment at the indicated times was analyzed by semi-quantitative PCR in $\mathrm{H} 1299$ cells (upper panels). Knockdown efficiency after $48 \mathrm{~h}$ was assessed by immunoblotting (lower panels). (d) Schematic showing human paox promoter with two potential AP-1-binding sites ( $\mathrm{P} 1$ and $\mathrm{P} 2$ ) predicted in silico (upper panel). The effect of overexpresssing c-Jun, JunB, FosB or the c-Jun/FosB and JunB/FosB dimers on the promoter activity of paox (left) or collagenase (right) was assessed by luciferase reporter assay in H1299 cells. RLU: relative luciferase units, which are ratios of luciferase and $\beta$-galactosidase activities. All values are means \pm S.D. of duplicate experiments. (e) The effect of ablating the predicted AP1-binding sites on paox promoter activity was assessed using the wild-type and mutated (Mutant P1 + P2) promoters (left panel) in the absence or presence of dominantnegative c-Jun (TAM67) or FosB (DNFosB) (right panel). All values are means \pm S.D. of duplicate experiments. (f) H1299 cells exposed to doxorubicin for the indicated time periods were used for chromatin immunoprecipitation (ChIP) using antibodies against the indicated AP-1 members (lower panel). Total DNA collected before immunoprecipitation was used as input control and binding specificity was demonstrated by the negative signal in anti-rabbit lgG control. Schematic shows sites of enrichment for AP-1 on the human paox promoter (upper panel)

(Figure 2d, lower panels). In order to locate the binding sites for AP-1 on the paox promoter, the two predicted repressor sites (P1 and P2) were mutated. As expected, mutations of both $\mathrm{P} 1$ and $\mathrm{P} 2$ sites increased the basal promoter activity by $>10$-fold relative to the wild-type paox promoter, indicating that $P 1$ and $P 2$ were indeed the repression sites of these selective AP-1 factors (Figure 2e, left panel). Furthermore, expression of transactivation-deficient mutants of c-Jun (TAM67) or FosB (DFosB), which exert a dominant negative effect on AP-1 transcriptional activity, ${ }^{29,30}$ led to the derepression of the paox promoter, which was not observed upon mutation of the predicted binding sites of AP-1 factors, $\mathrm{P} 1$ and P2 (Figure 2e, right panel). Moreover, the mutant promoter was not suppressed by the AP-1 members (Supplementary Figure 2A), altogether confirming the requirement of these sites for AP-1-mediated repression of paox.
To determine whether these three AP-1 transcription factors could bind to paox promoter in vivo, we performed chromatin immunoprecipitation (ChIP) of c-Jun, JunB and FosB on regions corresponding to $\mathrm{P} 1$ and $\mathrm{P} 2$. All three AP-1 members were highly enriched at the predicted sites (Figure 2f), but significant loading of JunB and FosB only occurred after doxorubicin treatment, whereas C-Jun was generally constitutively bound on P2. Altogether, the data show that selected members of the AP-1 family, namely c-Jun, JunB and FosB, bind to the paox promoter after genotoxic stress to repress its expression.

Selectivity in PAOX regulation by AP-1 members corresponds to DNp73 degradation. We next assessed the ability of the various members of the AP-1 family to degrade DNp73. Co-expression of DNp73 $\beta$ with several AP-1 
members showed that besides c-Jun, only JunB and FosB were equally competent at decreasing the steady-state levels of DNp73 (Figure 3a). This effect was further confirmed using the protein synthesis inhibitor cycloheximide $(\mathrm{CHX})$, which revealed that the half-life of $\mathrm{DNp} 73 \beta$ was significantly extended from $3.5 \mathrm{~h}$ to $\sim 6 \mathrm{~h}$ upon silencing the expression of FosB, JunB (Figure 3b) or c-Jun (Supplementary Figure 2B). Conversely, overexpression of these AP-1 members led to a significant reduction in the half-life of DNp73 (Supplementary Figures $2 \mathrm{C}$ and D). Furthermore, individual silencing of C-Jun, JunB or FosB in DNp73 $\beta$ inducible $\mathrm{H} 1299$ cells before doxorubicin treatment led to the stabilization of $\mathrm{DNp} 73 \beta$ levels at the basal level, and significantly inhibited the doxorubicin-mediated degradation of DNp73 $\beta$ (Figure 3c). We further also assessed whether JunB and FosB could degrade DNp73 independently of C-Jun. Overexpression of FosB or JunB, but not Fra1, in mouse embryonic fibroblasts deficient in c-Jun was able to degrade DNp73 $\beta$ to a similar extent as exogenously expressed c-Jun (Figure 3d), illustrating a clear functional redundancy of these three AP-1 members in regulating DNp73. These data together with the observation that only c-Jun, JunB and FosB can regulate paox expression (Figure 2), highlight the specificity of these AP-1 members in degrading DNp73 independently.
AP-1-mediated degradation of DNp73 occurs via the PAOX-Az pathway. As besides C-Jun, JunB and FosB also have the ability to downregulate paox and subsequently degrade DNp73 levels, it is highly likely that $A z 1(p)$ processing is a common mechanism regulated by all three AP-1 members. To test this hypothesis, we examined the effects of AP-1 members on Az1(p) production, with addition of putrescine being used as a positive control to induce $A z 1(p)$ (Figure $4 a$, last lane from left). Overexpression of c-Jun, JunB or FosB, but not Fra1, was able to upregulate $A z 1(p)$ expression, with no significant changes in the levels of $A z 1$ mRNA. To validate the role of $A z 1$ in mediating the degradation of DNp73 by JunB and FosB, we used the natural inhibitor of $A z 1, A Z I$, to block the function of this pathway. Degradation of DNp73 by JunB or FosB (Figure 4b), or C-Jun ${ }^{20}$ (data not shown) was significantly reduced in the presence of $A Z I$. Similarly, knockdown of $A z 1$ mRNA reduced $c$-Jun and FosB-mediated DNp73 degradation (Supplementary Figure 3a). Together, these data confirm the role of $A z 1$ as a downstream effector of all three AP-1 transcription factors to degrade DNp73.

Given that the production of $A z 1(p)$ is governed by polyamine biosynthesis, downregulation of catabolic enzyme PAOX by stress signals could lead to elevated polyamine content, thus enhancing $A z 1(p)$ production. Inhibition of
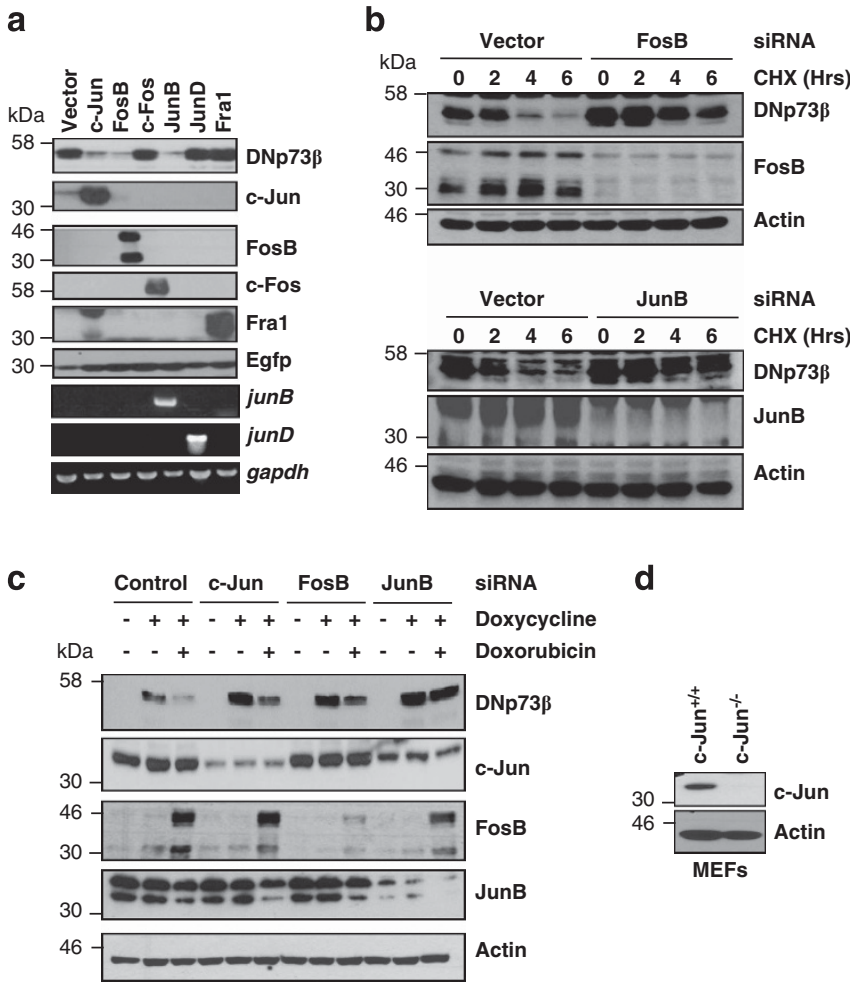

d

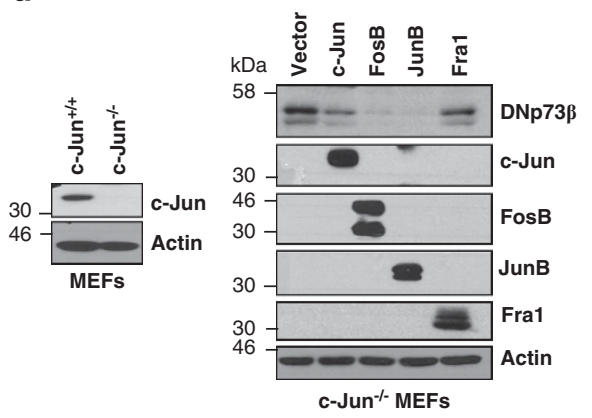

Figure 3 Selectivity in AP-1-mediated regulation of paox correlates with selectivity in AP-1-dependent DNp73 degradation. (a) Effect of co-expressing DNp73 $\beta$ with the indicated AP-1 members on the steady-state levels of DNp73 were assessed by immunoblotting $24 \mathrm{~h}$ after transfection in H1299 cells. Expression of AP-1 members was analyzed by immunoblotting and semi-quantitative PCR. Egfp was co-transfected as a control for transfection efficiency. (b) Half-life of transfected DNp73 $\beta$ after siRNAmediated knockdown of FosB and JunB were assessed by the addition of $25 \mu \mathrm{g} / \mathrm{ml}$ cycloheximide for the indicated times before immunoblotting. The adjacent graphs show the quantification of the remaining DNp73 (arbitrary values). (c) H1299 cells inducibly expressing DNp73 $\beta$ were transfected with control scramble, c-jun-, fosB-or junB-specific siRNA for $24 \mathrm{~h}$ before induction of DNp73 $\beta$ expression for a further $24 \mathrm{~h}$ by doxycycline addition. Cells were then treated with doxorubicin for $4 \mathrm{~h}$ and harvested for immunodetection. (d) $c$-jun ${ }^{-1-}$ mouse embryonic fibroblasts (MEFs) were transfected with DNp73 $\beta$ and the AP-1 members for $36 \mathrm{~h}$ before analysis (right panel). Status of endogenous c-Jun in wild-type and knockout MEFs is shown by immunoblotting (left panel) 
PAOX activity by treatment with MDL72527 indeed led to enhanced production of Az1(p) (Supplementary Figure 3B). We thus assessed whether suppression of paox is the mechanism employed by these three AP-1 factors to degrade DNp73. To verify this, PAOX was overexpressed to counteract the downregulation of endogenous paox mediated by c-Jun. Co-expression of Flag-tagged PAOX with c-Jun inhibited the reduction of DNp73 levels (Figure 4c), suggesting that transcriptional repression of paox is indeed mediating the effect of AP-1-dependent degradation of DNp73.

To further confirm the role of PAOX-Az axis in regulating DNp73, we examined whether Az1 was acting downstream of paox. Overexpression of $A Z I$, which inhibits $A z 1$, rescued DNp73 degradation caused by paox knockdown (Figure 4d). Similarly, silencing of Az1 partially inhibited DNp73 degradation induced by paox silencing (Figure 4e). Taken together, these data demonstrate that suppression of the polyamine catabolic enzyme paox by c-Jun, JunB and FosB leads to the degradation of DNp73 via the Az pathway.

PAOX inhibition reverses DNp73-dependent chemoresistance. Analysis of human cancer data sets using Oncomine showed that paox was indeed highly expressed in several types of tumors relative to their normal counterparts (Supplementary Figure 4A), emphasizing a potential clinical relevance of PAOX. Given that DNp73 overexpression leads to resistance to chemotherapeutic drugs such as cisplatin, ${ }^{5}$ and that PAOX expression regulates the abundance of $\mathrm{DNp} 73$, we assessed whether inhibition of PAOX with MDL72527 can reverse the chemoresistance mediated by DNp73. Treatment with MDL72527 was sufficient for the reduction of DNp73 $\beta$ levels in the SH-SY5Y neuroblastoma cell line overexpressing $\mathrm{DNp} 73 \beta$ in a dose-dependent manner (Supplementary Figure 4B). Concomitant cell death analysis revealed that cisplatin treatment resulted in increased cell death only in vector SHSY-5Y cells but not in DNp73 $\beta$ overexpressing cells ( $\%$ dead cells $0 / 10 / 20 \mu \mathrm{M}$ cisplatin - vector versus DNp73 $\beta \quad$ cells: 5.85/16.8/ 30.35 versus $4.8 / 3.35 / 2.65$; Figure $5 a)$. Treatment with MDL72527 alone caused a slight but consistent increase in cell death in SHSY5Y cells (\% dead vector cells \pm MDL72527: 5.85 versus 9.5). However, treatment of MDL72527 in combination with cisplatin potentiated cell death in SH-SY5Y-DNp73 $\beta$ (\% dead cells $0 / 10 / 20 \mu \mathrm{M}$ cisplatin - vector versus DNp73 $\beta$ cells: 10.6/32.1/30.2 versus 6.6/10.4/24.95; Figure 5a). Similar results were observed when the DNp73 $\beta$ overexpressing cells were treated with doxorubicin ( $\%$ dead cells 0/0.25/0.5/1 $\mu \mathrm{M}$ doxorubicin vector versus DNp73 $\beta$ cells: $2 / 22.6 / 29.45$ versus $2 / 1.45 / 1.1$ ), and the same potentiation effect of MDL72527 on cell death was observed specifically in DNp73 $\beta$ cells (\% dead cells

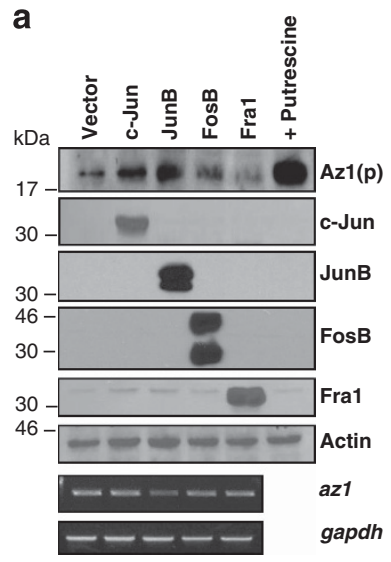

C

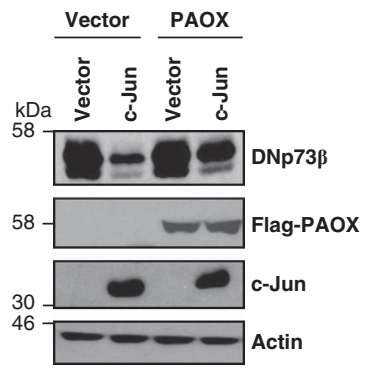

b

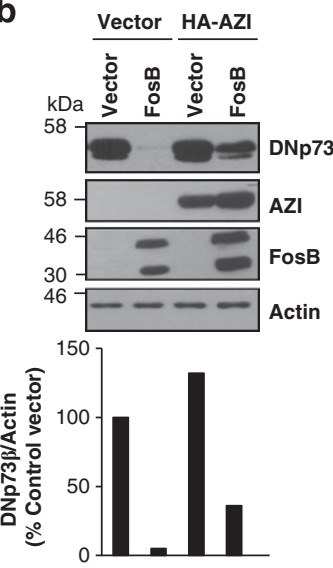

d

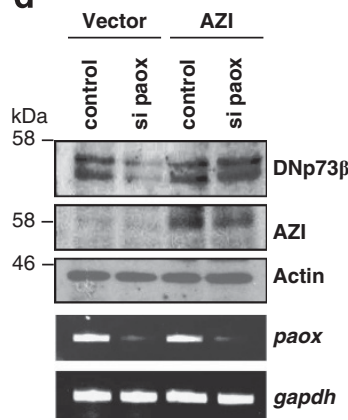

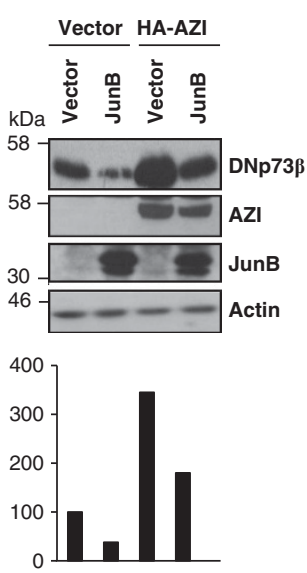

e

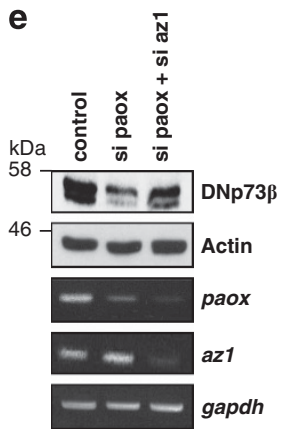

Figure 4 AP-1-mediated DNp73 depletion occurs via the PAOX-antizyme pathway. (a) Effect of overexpressing AP-1 members on the expression of Az1 mRNA and processed Az, [Az1 (p)], was assessed by semi-quantitative RT-PCR and immunodetection, respectively, $24 \mathrm{~h}$ after transfection in $\mathrm{H} 1299$ cells. (b and $\mathbf{c}$ ) Effect of the AP-1 members on transfected DNp73 $\beta$ in the presence of the naturally occurring antizyme inhibitor, AZl (b) or Flag-tagged PAOX (c) was determined by immunoblot analysis, $24 \mathrm{~h}$ after transfection in $\mathrm{H} 1299$ cells. Lower panels show quantification of DNp73 from the respective lanes from the blots above (b). (d and e) Effect of siRNA-mediated silencing of paox in the presence of AZI (d) or Az1 siRNA (e) on co-transfected DNp73 $\beta$ levels was determined by immunoblotting $24 \mathrm{~h}$ and $48 \mathrm{~h}$ after transfection in $\mathrm{H} 1299$ cells, respectively. Knockdown efficiency was determined by semi-quantitative RT-PCR 

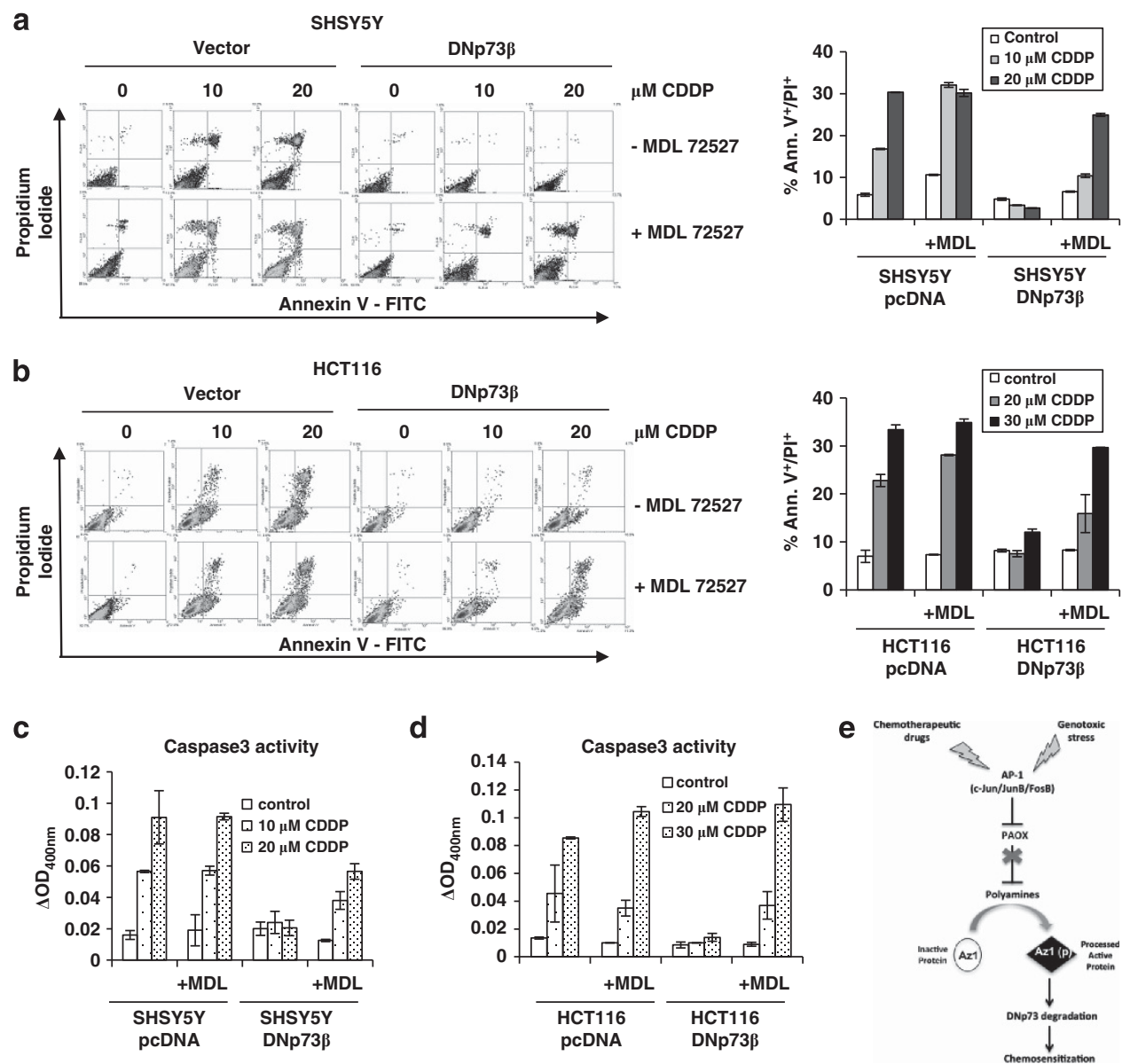

Figure 5 PAOX inhibition reverses chemoresistance by inducing apoptosis. (a and $\mathbf{b}$ ) SHSY5Y-DNp73 $\beta$ or SHSY5Y-pcDNA cells were treated with the indicated doses of cisplatin (CDDP) (a) in the absence or presence of $200 \mu \mathrm{M}$ of MDL72527 for $24 \mathrm{~h}$, and the proportion of cell death was determined by Annexin V/propidium iodide (PI) staining and flow cytometry. Raw data are shown as density plots (left) and percentage of Annexin $\mathrm{V}^{+} / \mathrm{PI}^{+}$cells are represented in a graphical format (right). The same experiment as a was repeated with HCT116-pcDNA and HCT-116-DNp73 $\beta$ (b). All values are means \pm S.D. of duplicate experiments. (c and d) SHSY5Y-DNp73 $\beta$ or SHSY5Y-pcDNA cells were treated with the indicated doses of cisplatin (CDDP) in the absence or presence of $200 \mu \mathrm{M}$ of MDL72527 for $24 \mathrm{~h}$ and caspase-3 activity in cell lysates were measured using a colorimetric protease assay. The values were calculated by subtracting background signal (with no substrate) from duplicate experiment (c). The same experiment as $\mathbf{c}$ was repeated in HCT116-pcDNA and HCT116-DNp73 $\beta$ cells (d). All values are means \pm S.D. of duplicate experiments. (e) Schematic illustrates the proposed mechanism by which genotoxic stresses can induce the degradation of anti-apoptotic DNp73 by activating c-Jun, FosB and JunB. Upon stress, these three AP-1 family members are induced and inhibit the expression of the polyamine catabolic enzyme paox, resulting in the eventual increase in the expression of Az1 (p), which leads to the degradation of DNp73, and consequently, chemosensitizes to genotoxic drugs

0/0.25/0.5/1 $\mu \mathrm{M}$ doxorubicin - with versus without MDL72527: 4.85/17/24 versus 2.75/4.75/17.25; Supplementary Figure 5A). Furthermore, resistance induced by stable DNp73 $\beta$ overexpression in another cell line, HCT116 (\% dead cells 0/20/30 $\mu \mathrm{M}$ cisplatin - vector versus DNp73 $\beta$ cells: 7/22.8/ 33.4 versus $8.2 / 7.55 / 12.05$ ) was equally reversed in the presence of MDL72527 (\% dead cells $0 / 20 / 30 \mu \mathrm{M}$ cisplatin vector versus $\mathrm{DNp} 73 \beta$ cells: $7.35 / 28.1 / 34.9$ versus $8.3 / 15.9 /$ 29.65; Supplementary Figure $5 B$ and Figure $5 b$ ). The chemosensitization effect of MDL72527 upon cisplatin or doxorubicin was verified to be through apoptotic activation as demonstrated by enhancement in cleaved-caspase-3 activity (Figures $5 \mathrm{c}$ and $d$, and Supplementary Figure $5 \mathrm{C}$ ). In addition, siRNA-mediated knockdown of paox before cisplatin treatment also led to sensitization to cell death in DNp73 $\beta$ overexpressing HCT116 (Supplementary Figure 5D). These data therefore indicate that the protective effect of DNp73 against exposure to chemotherapeutic drugs could be reversed by inhibition of the polyamine catabolic enzyme PAOX.

\section{Discussion}

High levels of DNp73 have been correlated with increased chemoresistance and poor clinical outcome in many cancers. ${ }^{8-10}$ Therefore, understanding mechanisms of how DNp73 expression is regulated in cancers and subsequently, devising strategies to specifically target DNp73 are critical for improving treatment. ${ }^{31}$ In this respect, we have previously shown that DNp73 is degraded by c-Jun upon exposure to genotoxic stress via the Az-mediated degradation pathway. ${ }^{20}$ However, the exact mechanisms for this process as well as its relevance to DNp73 in mediating chemoresistance was unclear. Az has been shown to degrade several cell-cycle regulatory proteins, including cyclin D1, Smad1 and Aurora A kinase, as well as Mps1, a protein that regulates centrosome 
duplication, ${ }^{32-35}$ supporting its role as an inhibitor of tumor cell proliferation and transformation. The expression of $\mathrm{Az}$ is tightly controlled at the post-transcriptional level by $a+1$ frameshift translation mechanism, which is regulated by the polyamine biosynthesis pathway. ${ }^{21}$ Polyamine homeostasis in cells is modulated by the interplay of several anabolic and catabolic enzymes ${ }^{22}$ and we have now shown that paox is specifically targeted by selected AP-1 factors that were activated upon exposure to chemotherapeutic drugs. PAOX is a FAD-dependent oxidase that preferentially retro-converts NASp to spermidine and $\mathrm{N}$-acetylspermidine to putrescine. ${ }^{22}$ Putrescine has been shown to be less effective at inducing $A z$ expression compared with spermidine and spermine ${ }^{36}$ and therefore inhibition of PAOX would likely lead to higher levels spermine and spermidine relative to putrescine, thus enabling a more pronounced induction of $A z$ in response to genotoxic stress. Many studies have shown that MDL72527, a chemical inhibitor of PAOX, can potentiate apoptosis in several cancer cells both in vitro and in vivo. ${ }^{37}$ Conversely, expression of another polyamine catabolic enzyme SSAT under the keratinocyte-specific $\mathrm{K} 6$ promoter was shown to promote skin tumors. ${ }^{38}$ Interestingly, treatment of these mice with MDL72527 partially counteracted tumor growth, ${ }^{38}$ indicating that inhibition of polyamine catabolism can be a potential therapeutic tool to suppress tumor formation. It is noteworthy that enhanced polyamine synthesis has also been shown to promote tumor growth and progression. ${ }^{38}$ For example, overexpression of the polyamine biosynthesis enzymes ODC or SAMDC induced malignant transformation. ${ }^{38,39}$ All these observations together suggest that disruption of the homeostatic balance of polyamine ratios and not their overall cellular increase could support tumorigenesis. Our current findings that inhibition of PAOX in DNp73-overexpressing cancer cells increases their sensitivity to apoptosis-inducing chemotherapeutic drugs are consistent with these observations. Interestingly, several studies targeting the polyamine pathways in conjunction with other drugs are currently in clinical trials. ${ }^{40}$ However, the direct role of PAOX in tumor formation in vivo and its effects on the levels of the different polyamines warrants further investigation in the future.

PAOX promoter is repressed upon exposure to genotoxic stresses through selective members of the AP-1 transcription family, namely c-Jun, JunB and FosB. c-Jun is known to have a dual role in regulating cell fate where it can either enable apoptotic responses or support growth and proliferation, depending on cellular context. ${ }^{41}$ These distinct functions of c-Jun might be determined by the interplay with additional factors such as the interaction with other AP-1 members. Similar to c-Jun, other AP-1 members can also be induced by DNA-damaging signals, ${ }^{42,43}$ indicating their potential involvement in promoting apoptosis with c-Jun. The data presented here show that C-Jun, JunB and FosB are activated by chemotherapeutic drugs to regulate DNp73 degradation. Although JunB is classically known to antagonize some biological functions of c-Jun such as cell proliferation, ${ }^{27}$ both also share redundancy on certain target genes, which include Egfr and Proliferin. ${ }^{44,45}$ On the other hand, FosB has been shown to bind in a complex with c-Jun, which was associated with FasL induction and cell death, indicating that C-Jun/FosB dimer has a pro-apoptotic function. ${ }^{46}$ Consistently, our data indicate that similar to C-Jun, expression of JunB and FosB could also induce the suppression of paox, increase the level of $\mathrm{Az}$ and lead to the subsequent degradation of DNp73. Moreover, JunB and FosB had similar effects in c-Jun-deficient cells, suggesting a level of in-built redundancy to perhaps ensure the efficient regulation of DNp73 levels upon genotoxic insults.

In conclusion, we have unraveled a mechanism through which selective AP-1 transcription factors c-Jun, JunB and FosB induce the degradation of anti-apoptotic DNp73 by Az, via the suppression of polyamine catabolic enzyme PAOX (Figure 5e). We have therefore identified PAOX as a positive regulator of DNp73 that defines a potential strategy to improve the therapeutic index of cytotoxic drugs in DNp73-overexpressing chemoresistant tumors.

\section{Materials and Methods}

Cells and reagents. Cell lines used are as follows: human cell lines - p53 null H1299 lung cancer cells - parental and with the inducible DNp73ß-constructtet-on system; p53 proficient neuroblastoma cell line SHSY5Y and colorectal cell line HCT116 stably expressing DNp73 $\beta$; mouse fibroblasts - wild-type and cJun $^{-1-} .2 \mu \mathrm{g} / \mathrm{ml}$ of doxycycline was added to H1299 and HCT116 DNp73 $\beta$ inducible cells to induce DNp73 $\beta$. Chemicals and inhibitors used: doxorubicin (Sigma, St. Louis, MO, USA), Cisplatin (Sigma), putrescine (Sigma), MDL72527 (Santa Cruz Biotechnology, Santa Cruz, CA, USA) and CHX (Sigma).

Plasmids, siRNAs and transfection. Plasmids used in this study are as follows: pCDNA3.1, DNp73 $\beta$, c-Jun, Flag-Tam67, JunB, FosB, Flag-Delta Fosb, c-Fos, Fra1, JunD, HA-AZI, Flag-PAOX (kind gift from Dr. Robert Casero Jr., John Hopkins University School of Medicine), $\beta$-gal, Egfp, pGL3-luc basic, various constructs of paox-luc (as indicated in the results section) and collagenase-luc. $2 \times 10^{5}$ cells were transfected with the various plasmids using Lipofectamine PlusReagent. Total amount of transfected DNA were equalized with appropriate amounts of pCDNA3 vector in all cases. Cells were collected $24-48 \mathrm{~h}$ after transfection for immunoblot analyses. siRNA transfections were performed using Transmessenger transfection kit (Qiagen, Hilden, Germany) or HiPerfect (Qiagen) and cells were harvested $48 \mathrm{~h}$ post transfection. Target sequence of siRNAs used are as follows: human c-jun: 5'-AAGAACGTGACAGATGAGCAG-3'; human junb: 5'-GAACGTGACAGATGAGCAG-3'; human fosB: $5^{\prime}$-GAGGAAGAGGAGAAG CGAA-3'; human paox. 5'-CGGAGATGATGGACACATA-3'; human Az1: $5^{\prime}$-GCTGAATGTAACAGAGGAA- $3^{\prime}$ and scramble control: 5' AATTCTCCGAA CGTGTCACGT $3^{\prime}$

Immunoblot analysis and half-life determination. Immunoblot analysis was performed essentially as described. ${ }^{47} \mathrm{~A}$ measure of $50 \mu \mathrm{g}$ cell lysate was used for analyses and detected using enhanced Chemiluminescent reagent (Amersham Biosciences, Buckinghamshire, UK) or Super signal West Dura (Pierce Biotechnology, Rockford, IL, USA). Antibodies used in this study are: anti-p73 (ER15; Calbiochem, San Diego, CA, USA), anti-p73 (GC15; Calbiochem), anti-HA (Y-11; Santa Cruz Biotechnology), anti-FLAG (M2; Stratagene, La Jolla, CA, USA), anti-c-Jun (Cell Signaling Technology, Danvers, MA, USA), anti-FosB (5G4; Cell Signaling Technology), anti-JunB (P169; Cell Signaling Technology), anti-Fra1 (R20; Santa Cruz Biotechnology), anti-Actin (Sigma), anti-Egfp (Santa Cruz Biotechnology), anti-cyclin D1 (H295; Santa Cruz Biotechnology), anti-Az1(p) (a gift from Dr. John Mitchell, Northern Illinois University), anti-AZI (a gift from Dr. Senya Matsufuji, The Jikei University School of Medicine). For measuring half-life of proteins, cells were treated with $50 \mu \mathrm{g} / \mathrm{ml}$ of $\mathrm{CHX}$ and collected at indicated time points for immunoblotting.

Semi-quantitative RT-PCR analyses. Total RNA was prepared from cells using TRIZOL reagent (Invitrogen, Carlsbad, CA, USA) and converted into single-strand cDNA using Superscript II Reverse Transcriptase (Invitrogen). Semiquantitative PCR was performed using Taq polymerase (Qiagen) in a $25-\mu$ l solution according to manufacturers' protocols. The reactions were carried under the following conditions: initial $94^{\circ} \mathrm{C}$ for $3 \mathrm{~min}, 94^{\circ} \mathrm{C}$ for $35 \mathrm{~s}, 55^{\circ} \mathrm{C}$ for $35 \mathrm{~s}$ and $72{ }^{\circ} \mathrm{C}$ for $35 \mathrm{~s}$ for indicated cycles. Primers specific to both human $(\mathrm{h})$ and mouse $(\mathrm{m})$ genes are: 
samdc (h) forward 5'GGTGATGGAAGCTGCACATTT3', reverse 5'-GGTGGT ACCACATGTCTTCAA-3'; odc (h) forward 5'-GACTGAAATACAGTTGGTG-3', reverse $5^{\prime}$-TCAGATCCAGGAAAGCCAC- $3^{\prime}$; spds (h) forward $5^{\prime}$-GAGTTCTCC TACCAGGAGATG-3', reverse 5'-GTAATAGGACTCCTTGAAGAG-3'; spms (h) forward 5'-GTGTTCTGGACCTTCAGAG-3', reverse 5'-AAACTGCTTCGAGTG TAG-3'; paox (h) forward 5'-AAGCAATCAGGAAGATAGGC-3', reverse 5'-CAGAAGTACTTCTTCATCCG-3', ssat (h) forward 5'-GAAGAGGTGCTTCT GATCTGTC-3', reverse $5^{\prime}$-ACTCTCCAAACTGCATCTGAG-3'; fosb (h) forward 5'-CAAGACTTGGAAACTTGATTG-3', reverse 5'-TACAGAATCAGCTTCAGC TAC-3'; gapdh (m/h) forward 5'-CATTGACCTCAACTACATG-3', reverse 5'-CTTCTCCATGGTGGTGAAG-3'; cyclin D1 (h) forward 5'-CTGGCCATGAAC TACCTGGA-3', reverse $5^{\prime}$-GTCACACTTGATCACTCTGG- $3^{\prime}$; cjun $(\mathrm{m} / \mathrm{h})$ forward 5'-CTGCTCGTCGGTCACGTTCTTGGG-3', reverse $5^{\prime}$-ATGCCCTCAACGCCTC GTTCCTCC-3'; junb (m) forward 5'-ACCTGGCGGATCCCTATCGG-3', reverse 5'-GCTCCGGACCAGCATAGACG-3'; fra1 (m/h) forward 5'- GGCTGCAGCGAGA GATTGA-3', reverse $5^{\prime}$-TGGTCATGAGTGTGGGGGT-3'; cfos (m/h) forward 5'-CCAACCTGCTGAAGGAGAAG-3', reverse 5'-GCTGCTGATGCTCTTGACAG-3'; junD $(\mathrm{m})$ forward 5'-GATTGAAACCAGGGTCGG-3', reverse 5'-CTGCTCAGGTTG GCGTAGAC-3'.

Site-directed mutagenesis (SDM) and luciferase reporter assays. The -3045 to -997 paox promoter fragment was cloned into in pGL3 basic or enhancer vector (Promega, Fitchburg, WI, USA). H1299 cells were transiently transfected with $0.2 \mu \mathrm{g}$ of the relevant plasmids along with the reporter (paox-luc or collagenase-luc) and $\beta$-galactosidase constructs to normalize for transfection efficiency. Luciferase assays were performed as described. ${ }^{47}$

Potential AP-1-binding sites were found using TESS (Transcription Element Search System online software). SDM of the AP-1 sites $\mathrm{P} 1$ and $\mathrm{P} 2$ were carried out as described. ${ }^{5}$ Primers used are: SDM-P1 $5^{\prime}$-ATGAACAAGCCAAGTTACAGT CAAAGCCAC-3'; SDM-P2 5'-ACCGCCCAGAGAATAAGCCCGACTC-3'.

ChIP. H1299 cells treated with $5 \mu \mathrm{M}$ doxorubicin for 0,3 and $6 \mathrm{~h}$ were crosslinked with $1 \%$ formaldehyde for $10 \mathrm{~min}$ at room temperature. The reaction was stopped by the addition of ice-cold PBS and washed twice with the same buffer. Cells were lysed for $10 \mathrm{~min}$ at $4{ }^{\circ} \mathrm{C}$ with Triton X-100 lysis buffer $(0.25 \%$ triton X-100, $10 \mathrm{~mm}$ EDTA, $10 \mathrm{mM}$ Tris $\mathrm{pH} 8,100 \mathrm{mM} \mathrm{NaCl}$ and protease inhibitor cocktail (Roche Applied Science, Mannheim, Germany). Samples were centrifuged at 1500 r.p.m. at $4{ }^{\circ} \mathrm{C}$ for $5 \mathrm{~min}$ and the pellet containing the chromatin fraction was lysed in SDS lysis buffer (1\% SDS, $5 \mathrm{mM}$ EDTA, $50 \mathrm{mM}$ Tris pH8 and protease inhibitor cocktail). Chromatin extracts containing genomic DNA were sonicated on ice to an average size of $500 \mathrm{bp}$ and subsequently immunoprecipitated using control anti-IgG, anti c-Jun, anti-FosB or anti-JunB (all from Cell Signalling as previously described) at $4^{\circ} \mathrm{C}$. The immunocomplex was incubated with $50 \mu$ l of $50 \%$ slurry Protein A-Sepharose beads (Invitrogen) for a further $2 \mathrm{~h}$ at $4{ }^{\circ} \mathrm{C}$. Samples were washed with, $1 \times$ HS buffer $(0.1 \%$ SDS, $1 \%$ Triton X-100, 2 mM EDTA, 20 mM Tris-HCl, pH 8, 500mM NaCl), $1 \times$ LS buffer (0.1\% SDS, $1 \%$ Triton X-100, $2 \mathrm{mM}$ EDTA, $20 \mathrm{mM}$ Tris-HCl, pH 8, $150 \mathrm{mM} \mathrm{NaCl}), 1 \times 250 \mathrm{mM}$ $\mathrm{LiCl}$ buffer at $4{ }^{\circ} \mathrm{C}$ for $10 \mathrm{~min}$, followed by $2 \times$ in TE buffer. DNA/protein complex was eluted three times with $100 \mu \mathrm{l}$ of extraction buffer (1\%SDS and $100 \mathrm{mM}$ $\mathrm{NaHCO}_{3}$ ) for $15 \mathrm{~min}$. To reverse DNA/protein crosslink, eluted samples were incubated with $200 \mathrm{mM} \mathrm{NaCl}$ for $4 \mathrm{~h}$ at $65^{\circ} \mathrm{C}$, followed by proteinase $\mathrm{K}$ treatment at final concentration of $500 \mu \mathrm{g} / \mathrm{ml}$ at $45^{\circ} \mathrm{C}$ for $2 \mathrm{~h}$. DNA was purified for PCR using DNA Qiaquick columns (Qiagen).

Semi-quantitative PCR analyses were performed to assess the ChIP-enriched DNA, and non-immunoprecipitated input DNA was included as loading control. The following primers were used: $\mathrm{P1}$ paox promoter forward $5^{\prime}$-GGAGAAGTCTTAC AGCATGAT-3', P1 paox promoter reverse 5'- ACAAGGTATGTGCTTCTCCTA-3'; P2 paox promoter forward $5^{\prime}$ - TCCTTAGTCCTGGCATGTCGG-3', P2 paox promoter reverse 5'- GTGACCTGGTGTCGCTGGATT-3'.

Cell death assay. Cell death was measured by propidium iodide $(\mathrm{PI})$ exclusion assay. SHSY5Y stably transfected with pcDNA or DNp73 $\beta$ cells were treated with indicated doses of doxorubicin or cisplatin with or without MDL72527. Both floating and attached cells were harvested and washed $2 \times$ with PBS before re-suspension in $2 \mu \mathrm{g} / \mathrm{ml} \mathrm{PI}$. Percentage of Annexin $\mathrm{V}^{+} / \mathrm{PI}^{+}$cells was measured by flow cytometry (BD Biosciences, FACScalibur, San Jose, CA, USA).

Polyamine measurements. Polyamine levels were measured by HPLC as described previously. ${ }^{48}$ Cells treated with doxorubicin or cisplatin, or untreated controls $\left(6 \times 10^{5}\right.$ cells) were harvested and polyamines were extracted from cell lysate with $5 \%$ trichloroacetic acid and the supernatant after centrifugation at $27000 \times g$ for $15 \mathrm{~min}$ at $4{ }^{\circ} \mathrm{C}$ was used for polyamine measurement by HPLC. The precipitate was used for measurement of protein content.

Caspase activity assay. Caspase- 3 activity was measured by colorimetric assay according to manufacturer's manual (ApoTarget Caspase-3 Kit, Life Sciences, Invitrogen). In brief, cells were lysed in Tris-buffered saline containing reagent and protein concentrations were measured using Comassie Bradford's reagent. $200 \mu \mathrm{g}$ of the extracted protein were mixed with $2 X$ extraction buffer and $200 \mu \mathrm{M}$ of DEVD-pNA caspase-3 substrate and incubated for $2 \mathrm{~h}$ away from light at $37^{\circ} \mathrm{C}$. Developed reaction was measured at the optical density of $400 \mathrm{~nm}$ using spectrophotometry, and the absorbance reading for caspase activity was calculated by subtracting the absorbance of blank samples (without substrate).

Acknowledgements. We thank the National Medical Research Council of Singapore for financial support to KS and Drs John Mitchell, Senya Matsufuji, Robert Casero Jr. and Leah Vardy for reagents and advise on their use.

1. Melino G, De Laurenzi V, Vousden KH. p73: friend or foe in tumorigenesis. Nat Rev Cancer 2002; 2: 605-615; Review.

2. Jost CA, Marin MC, Kaelin Jr WG. p73 is a simian [correction of human] p53-related protein that can induce apoptosis. Nature 1997; 389: 191-194.

3. Stiewe T, Theseling CC, Pützer BM. Transactivation-deficient Delta TA-p73 inhibits p53 by direct competition for DNA binding: implications for tumorigenesis. J Biol Chem 2002; 277: $14177-14185$.

4. Liu G, Nozell S, Xiao H, Chen X. DeltaNp73beta is active in transactivation and growth suppression. Mol Cell Biol 2004; 24: 487-501.

5. Toh WH, Logette E, Corcos L, Sabapathy K. TAp73beta and DNp73beta activate the expression of the pro-survival caspase-2S. Nucleic Acids Res 2008; 36: 4498-4509.

6. Tanaka Y, Kameoka M, Itaya A, Ota K, Yoshihara K. Regulation of HSF1-responsive gene expression by N-terminal truncated form of p73alpha. Biochem Biophys Res Commun 2004; 317: 865-872.

7. Wilhelm MT, Rufini A, Wetzel MK, Tsuchihara K, Inoue S, Tomasini $\mathrm{R}$ et al. Isoform-specific p73 knockout mice reveal a novel role for delta Np73 in the DNA damage response pathway. Genes Dev 2010; 24: 549-560.

8. Zaika Al, Slade N, Erster SH, Sansome C, Joseph TW, Pearl M et al. DeltaNp73, a dominant-negative inhibitor of wild-type p53 and TAp73, is up-regulated in human tumors. J Exp Med 2002; 196: 765-780.

9. Concin N, Becker K, Slade N, Erster S, Mueller-Holzner E, Ulmer H et al. Transdominant DeltaTAp73 isoforms are frequently up-regulated in ovarian cancer. Evidence for their role as epigenetic p53 inhibitors in vivo. Cancer Res 2004; 64: 2449-2460.

10. Buhlmann S, Pützer BM. DNp73 a matter of cancer: mechanisms and clinical implications. Biochim Biophys Acta 2008; 1785: 207-216.

11. Lin KW, Nam SY, Toh WH, Dulloo I, Sabapathy K. Multiple stress signals induce p73beta accumulation. Neoplasia 2004; 6: 546-557.

12. Di C, Yang L, Zhang H, Ma X, Zhang X, Sun $\mathrm{C}$ et al. Mechanisms, function and clinical applications of DNp73. Cell Cycle 2013; 12: 1861-1867.

13. Stiewe T, Pützer BM. Role of $p 73$ in malignancy: tumor suppressor or oncogene? Cell Death Differ 2002; 9: 237-245.

14. Maisse C, Munarriz E, Barcaroli D, Melino G, De Laurenzi V. DNA damage induces the rapid and selective degradation of the DeltaNp73 isoform, allowing apoptosis to occur. Cell Death Differ 2004; 11: 685-687.

15. Rossi M, De Laurenzi V, Munarriz E, Green DR, Liu YC, Vousden KH et al. The ubiquitin-protein ligase Itch regulates p73 stability. EMBO J 2005; 24: 836-848.

16. Tsai KK, Yuan ZM. c-Abl stabilizes $p 73$ by a phosphorylation-augmented interaction. Cancer Res 2003; 63: 3418-3424.

17. Bernassola F, Salomoni P, Oberst A, Di Como CJ, Pagano M, Melino G et al. Ubiquitin-dependent degradation of p73 is inhibited by PML. J Exp Med 2004; 199: 1545-1557.

18. Asher G, Tsvetkov P, Kahana C, Shaul Y. A mechanism of ubiquitin-independent proteasomal degradation of the tumor suppressors p53 and p73. Genes Dev 2005; 19: 316-321.

19. Ohtsuka T, Ryu H, Minamishima YA, Ryo A, Lee SW. Modulation of p53 and p73 levels by cyclin G: implication of a negative feedback regulation. Oncogene 2003; 22: 1678-1687.

20. Dulloo I, Gopalan G, Melino G, Sabapathy K. The antiapoptotic DeltaNp73 is degraded in a c-Jun-dependent manner upon genotoxic stress through the antizyme-mediated pathway. Proc Natl Acad Sci USA 2010; 107: 4902-4907.

21. Matsufuji S, Matsufuji T, Miyazaki $Y$, Murakami $Y$, Atkins JF, Gesteland RF et al. Autoregulatory frameshifting in decoding mammalian ornithinedecarboxylase antizyme. Cell 1995; 80: 51-60.

22. Casero Jr RA, Marton LJ. Targeting polyamine metabolism and function in cancer and other hyperproliferative diseases. Nat Rev Drug Discov 2007; 6: 373-390. 
23. Shaulian E, Karin M. AP-1 in cell proliferation and survival. Oncogene 2001; 20 2390-2400.

24. Hess J, Angel P, Schorpp-Kistner M. AP-1 subunits: quarrel and harmony among siblings. J Cell Sci 2004; 117: 5965-5973.

25. Chinenov Y, Kerppola TK. Close encounters of many kinds: Fos-Jun interactions that mediate transcription regulatory specificity. Oncogene 2001; 20: 2438-2452.

26. Bakiri L, Matsuo K, Wisniewska M, Wagner EF, Yaniv M. Promoter specificity and biological activity of tethered AP-1 dimers. Mol Cell Biol 2002; 22: 4952-4964.

27. Eferl R, Wagner EF. AP-1: a double-edged sword in tumorigenesis. Nat Rev Cancer 2003; 3: 859-868.

28. Coffino P. Regulation of cellular polyamines by antizyme. Nat Rev Mol Cell Biol 2001; 2 188-194.

29. Young MR, Li JJ, Rincón M, Flavell RA, Sathyanarayana BK, Hunziker R et al. Transgenic mice demonstrate AP-1 (activator protein-1) transactivation is required for tumor promotion. Proc Natl Acad Sci USA 1999; 96: 9827-9832.

30. Skinner M, Qu S, Moore C, Wisdom R. Transcriptional activation and transformation by FosB protein require phosphorylation of the carboxyl-terminal activation domain. Mol Cell Biol 1997; 17: 2372-2380.

31. Irwin MS, Kondo K, Marin MC, Cheng LS, Hahn WC, Kaelin Jr WG. Chemosensitivity linked to p73 function. Cancer Cell 2003; 3: 403-410.

32. Newman RM, Mobascher A, Mangold U, Koike C, Diah S, Schmidt M et al Antizyme targets cyclin D1 for degradation. A novel mechanism for cell growth repression. J Biol Chem 2004; 279: 41504-41511.

33. Lin Y, Martin J, Gruendler C, Farley J, Meng X, Li BY et al. A novel link between the proteasome pathway and the signal transduction pathway of the bone morphogenetic proteins (BMPs). BMC Cell Biol 2002; 3: 15

34. Lim SK, Gopalan G. Antizyme1 mediates AURKAIP1-dependent degradation of Aurora-A Oncogene 2007; 26: 6593-6603.

35. Kasbek $\mathrm{C}$, Yang $\mathrm{CH}$, Fisk HA. Antizyme restrains centrosome amplification by regulating the accumulation of Mps1 at centrosomes. Mol Biol Cell 2010; 21: 3878-3889.

36. Yuan Q, Ray RM, Viar MJ, Johnson LR. Polyamine regulation of ornithine decarboxylase and its antizyme in intestinal epithelial cells. Am J Physiol Gastrointest Liver Physiol 2001; 280: G130-G138.
37. Seiler N, Renault J, Gossé F, Roussi S, Raul F. Cytotoxicity of the polyamine oxidase inactivator MDL 72527 to cancer cells: comparison with a saturated structural analogue. Int J Oncol 2005; 27: 1669-1676.

38. Wang X, Feith DJ, Welsh P, Coleman CS, Lopez C, Woster PM et al. Studies of the mechanism by which increased spermidine/spermine N1-acetyltransferase activity increases susceptibility to skin carcinogenesis. Carcinogenesis 2007; 28: 2404-2411.

39. Chen Y, Megosh LC, Gilmour SK, Sawicki JA, O'Brien TG. K6/ODC transgenic mice as a sensitive model for carcinogen identification. Toxicol Lett 2000; 116: 27-35

40. Nowotarski SL, Woster PM, Casero Jr RA. Polyamines and cancer: implications for chemotherapy and chemoprevention. Expert Rev Mol Med 2013; 15: e3.

41. Behrens A, Sibilia M, Wagner EF. Amino-terminal phosphorylation of c-Jun regulates stress-induced apoptosis and cellular proliferation. Nat Genet 1999; 21: 326-329.

42. Shaulian E, Karin M. AP-1 as a regulator of cell life and death. Nat Cell Biol 2002; 4: E131-E136; Review.

43. Hazzalin CA Le Panse $R$, Cano $E$ Mahadevan LC. Anisomycin selectively desensitizes signalling components involved in stress kinase activation and fos and jun induction. Mol Cell Biol 1998; 18: 1844-1854.

44. Zenz $R$, Scheuch $H$, Martin $P$, Frank $C$, Eferl $R$, Kenner $L$ et al. c-Jun regulates eyelid closure and skin tumor development through EGFR signaling. Dev Cell 2003; 4: 879-889.

45. Toft DJ, Rosenberg SB, Bergers G, Volpert O, Linzer DI. Reactivation of proliferin gene expression is associated with increased angiogenesis in a cell culture model of fibrosarcoma tumor progression. Proc Natl Acad Sci USA 2001; 98: 13055-13059.

46. Baumann S, Hess J, Eichhorst ST, Krueger A, Angel P, Krammer PH et al. An unexpected role for FosB in activation-induced cell death of T cells. Oncogene 2003; 22: $1333-1339$.

47. Toh WH, Siddique MM, Boominathan L, Lin KW, Sabapathy K. c-Jun regulates the stability and activity of the p53 homologue, p73. J Biol Chem 2004; 279: 44713-44722.

48. Igarashi K, Kashiwagi K, Hamasaki H, Miura A, Kakegawa T, Hirose S et al. Formation of a compensatory polyamine by Escherichia coli polyamine-requiring mutants during growth in the absence of polyamines. J Bacteriol 1986; 166: 128-134.

\section{Supplementary Information accompanies this paper on Cell Death and Differentiation website (http://www.nature.com/cdd)}

